\begin{tabular}{l|l} 
Exeview & TRENDS in Microbiology Vol.12 No.5 May 2004 \\
ELEVIER &
\end{tabular}

\title{
Drosophila: a polyvalent model to decipher host-pathogen interactions
}

\author{
Nicolas Vodovar, Carlos Acosta, Bruno Lemaitre and Frédéric Boccard
}

Centre de Génétique Moléculaire (CNRS UPR2167), Gif sur Yvette, F-91198, France

\begin{abstract}
In the past few years, several genetically amenable organisms have been used as models for the analysis of host-pathogen interactions. Among them, the fruit fly Drosophila melanogaster has been useful in elucidating signaling pathways and mechanisms that are used by the host to prevent and combat microbial infections. In addition, this model has also been used to identify virulence factors of opportunistic pathogens. Finally, the characterization of naturally infectious pathogens for Drosophila has illustrated its ability to activate immune responses that are adapted to its aggressors and highlights the potential of these flies to disseminate bacterial pathogens. Altogether, these approaches will allow the mechanisms involved in microbial infection and host defense responses to be dissected using genetic and genomic approaches.
\end{abstract}

Host-pathogen interactions are multi-step processes that involve several factors in both partners. In microbes, this complex process involves adhesion to the host surface and eventually leads to colonization and persistence, which provokes tissue damage and disease; these steps involve at least two sets of so-called virulence genes. The first subset of genes comprises those involved in the regulation of the adhesion process, which involves binding of microbial ligands to host receptors [1]. This molecular interaction is highly specific and has been shown to define the host range of several pathogens [2]. The target cells or organs are defined by the expression pattern of the receptors and their accessibility to the pathogen. The second subset of genes is mostly involved in adaptation and survival of the microbe within the host's hostile environment. In the host, defense mechanisms are activated following infection and eventually permit clearance of the microbe.

\section{Modeling host-pathogen interactions}

To study most processes involved in host-pathogen interactions a system must be set up that includes both the host and pathogen. Because many pathogens being investigated infect humans, surrogate model hosts that are amenable to biological analysis are required for their study. In vitro models devised on the basis of cultured-cell approaches are very useful, especially to identify host receptors or to dissect the intracellular fate of invasive bacteria. However, in vivo surrogate model hosts are preferred to in vitro models because they are more relevant

Corresponding authors: Bruno Lemaitre (lemaitre@cgm.cnrs-gif.fr), Frédéric Boccard (boccard@cgm.cnrs-gif.fr). to the natural disease process. In these in vivo models, the infectious agent should have the same tropism, and the interaction that takes place the same outcome, as occurs in the normal host. Most pathogens usually colonize a restricted number of hosts, thereby limiting the number of models that are available. To overcome this limitation, several strategies have been adopted; one possibility is to study genetically related pathogens that are naturally infectious in laboratory models (e.g. infection of mice by Salmonella enterica serotype Typhimurium). Another strategy is to use artificial routes of infection (e.g. intraperitoneous or intravenous injection) or to use a permissive developmental stage in 'non-host' models (e.g. suckling mouse model for Vibrio cholerae [3]). A third approach has made use of genetically modified animals that express a human receptor for a specific pathogen [2]. However, this latter approach relies on knowledge of the human receptor, which remains to be identified for several pathogens (e.g. Salmonella enterica serotypes).

Genetic analysis is the method chosen by most to dissect the mechanisms involved in complex host-pathogen interactions. It allows the identification of host and bacterial factors through genome-wide screening of genetic variants. Several methodologies have been set up to generate large numbers of bacterial variants. However, despite the availability of mammalian host models, the number of animals that can be used is limited. To overcome this problem, methodologies have been developed that involve screening a relatively large pool of bacterial variants for attenuated virulence phenotypes [4,5]. These methods allow the identification of genes that are required for in vivo virulence; these genes can then be further tested individually. Ideally, the host should also be amenable to genetic analysis. Although mammalian models are amenable to reverse genetics, identification of genes through forward genetics remains a challenge. Therefore, non-mammalian models, such as the mustard weed Arabidopsis thaliana [6,7], the nematode Caenorhabditis elegans [8,9], the social amoeba Dictyostelium discoideum [10,11] and the fruit fly Drosophila melanogaster [12,13], have been used as host models because they are easily amenable to both forward and reverse genetics. Their affordability and short generation time make them suitable models to carry out intensive analysis and therefore provide an appropriate alternative to mammalian models when characterizing the interplay between hosts and pathogens. Other insect models that lack tools for genetic analysis have also been developed, for example, 
the larger size of the greater wax moth Galleria mellonella facilitates a biochemical approach [14-16]. The use of these models has revealed a good correlation between pathogenesis in mammals and in lower organisms [17]. In this review, we describe in further detail different features of Drosophila-pathogen interactions that will probably improve our understanding of host-pathogen interactions.

\section{Drosophila as a model to characterize the complex host response \\ Drosophila immunity}

Drosophila has developed effective defense mechanisms to protect itself from overwhelming infection in the microorganism-enriched environments in which it spends its entire life. First, the entire body of Drosophila is encompassed by a cuticle that prevents microbial penetration. Surface epithelia that are found in areas including the trachea and gut, which are major routes of infection, are also lined with chitinous membrane to prevent direct contact between cells and microorganisms. To avoid colonization by ingested microbes, a hostile environment is maintained in the gut through low $\mathrm{pH}$ and constitutive secretion of antimicrobial agents (e.g. lysozyme) [12,18].

When physical barriers are accidentally or experimentally breached, the introduction of microbes within the body cavity activates a strong inducible immune response. Because Drosophila has been the favorite model over the past 90 years for deciphering developmental and cellular processes, numerous genetic and genomic tools are available for this system (Box 1). The availability of such tools and various infection methods has facilitated a detailed description of these inducible defense mechanisms.
The Drosophila immune response consists of both cellular and humoral responses. Cellular responses mainly involve professional macrophages termed plasmatocytes, which engulf incoming bacteria through phagocytosis [12,18]. Microbes also induce a strong systemic humoral response. Cuticle breakage leads to the rapid activation of proteolytic cascades that provoke coagulation and melanization in an attempt to restrict microbial spreading within the organism. Moreover, numerous immune effectors are secreted by the fat-body into the blood. Expression of the gene that encodes these factors is under the control of two parallel nuclear factor (NF)- $\mathrm{B}$ signaling pathways designated Toll and IMD (immune deficiency), which share strong similarities with those involved in mammalian innate immune responses (Figure 1) [12,18]. A repertoire of effectors, the expression of which is regulated by the Toll and IMD pathways, has been analyzed using microarray experiments [19-22]; for further information, see the Drosophila Immune Regulated Genes website (http://www. cnrs-gif.fr/cgm/immunity/drosophila_immunity_genes.html). Among these effectors are antimicrobial peptides, the expression of which is specified by the class of the infecting microbe. The IMD pathway is activated predominantly by Gram-negative bacteria, and in turn activates the expression of antibacterial peptide-encoding genes (e.g. diptericin), whereas the Toll pathway is predominantly activated by Gram-positive bacteria and fungi, and regulates the expression of genes that encode antifungal peptides (e.g. drosomycin) and also a subset of antibacterial peptides $[12,18]$. The expression of these two pathways can be monitored in vivo using reporter constructs [e.g. effector - GFP (green fluorescent protein) fusions]

\section{Box 1. Overview of Drosophila melanogaster genetics}

In Drosophila, as in many other organisms, mutations can be generated using chemical or physical agents. If chemical agents generate point mutations, ionizing radiations usually generate deletions of various sizes that constitute a good tool to be used to define a physical area in which the mutation is located. Transposon-mediated mutagenesis has also been greatly emphasized because it allows the generation of different types of mutants (disruption or deregulation of gene expression). Moreover, $P$ element tansposons can be remobilized, inducing small deletions by imprecise excision. Directed mutations can also be generated using homologous recombination [50]. Interestingly, mutations are easily tractable through generations using modified chromosomes, known as balancer chromosomes, which prevent meiotic recombination. These chromosomes are associated with phenotypic markers that allow genotypes of interest to be identified.

Synthetic Pelements are extensively used as a vector for transgenesis [51] and constitute a powerful tool for gene expression management. The yeast UAS-GAL4 system is fully functional in Drosophila when carried as transgenes [52], and therefore it has been largely used as an inducible system to express the gene of interest in a spatio-temporal manner [53]. It involves binding of the GAL4 transcriptional activator to UAS sequences, inducing gene expression downstream of these sequences. The generation of such individuals can be easily achieved by crossing transgenic individuals carrying a UAS-transgene with transgenic individuals expressing a GAL4 driver. Depending on the promoter sequence driving the expression of the GAL4 gene, the transgene can therefore be expressed in any tissue or cell type and at any time.

Similarly, the UAS-GAL4 system can be used to drive expression of dsRNA that induces degradation of the mRNA of the target gene, which has a role in promoting gene silencing [54]. In contrast to mammals, dsRNA does not provoke general protein synthesis blockage in Drosophila. It is therefore possible to clone large fragments of coding sequence as inverted repeats in a UAS expression vector to silence the expression of a specific target gene [54]. Even though RNA interference (RNAi) only generates hypomorphic mutants, it allows the study of genes that, when mutated, are associated with lethal phenotypes. It also permits the contribution of gene mutations in the pleiotropic phenotype to be identified in restricted cell types. The study of early lethal mutations can also be achieved by generating clones of cells that are homozygous for a mutation in an overall heterozygous individual. These clones can be obtained by forcing mitotic crossing-over that is mediated by expressing a site-specific recombinase (e.g. FLP-FRT system [55]).

Finally, the Drosophila genome sequence was completed a few years ago [56] and its annotation is under constant refinement [57]. The Genome Annotation Database (http://flybase.net/annot/) contains information about all the genes annotated, including their description, expression profile and mutant availability. In particular, this website gives access to large collections of mutants and transgenic lines that are available in Drosophila genetic centers. To date, the Drosophila genome is probably one of the most fully annotated eukaryotic genomes to be found in a database. The availability of these data has allowed the construction of DNA chips, allowing genome-wide analysis of different processes and a protein interaction map of the Drosophila proteome to be produced [58]; further information can be found at the Drosophila Interaction Database (http://portal.curagen.com/cgi-bin/interaction/ flyHome.pl). 


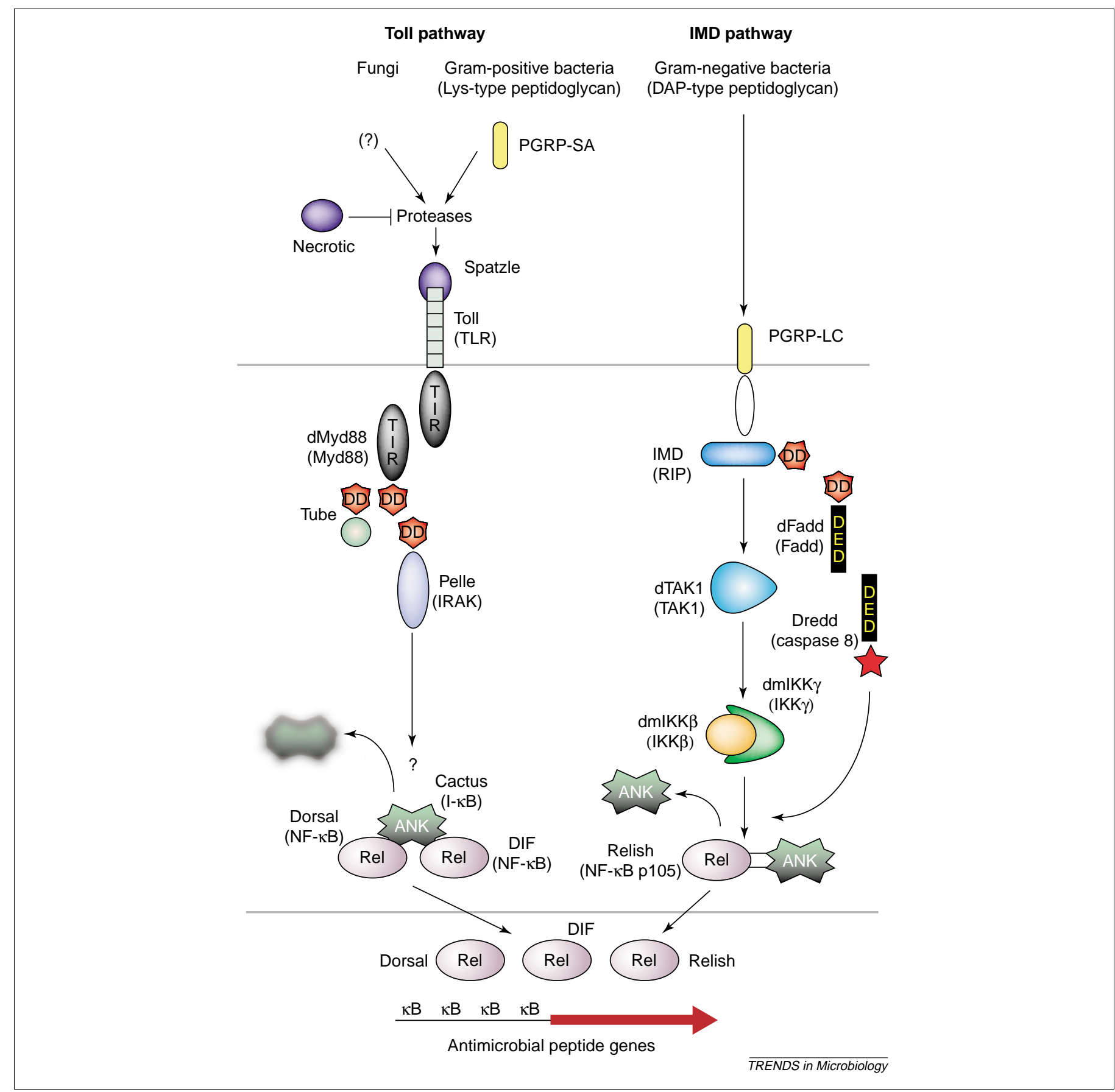

Figure 1. Drosophila IMD (immune deficiency) and Toll pathways are predominantly activated by Gram-negative bacteria and by Gram-positive bacteria or fungi, respectively. These two pathways allow Drosophila to adapt its immune response to the class of the invading microbe. The IMD and Toll pathways display strong similarities with mammalian TNFR (tumor necrosis factor receptor) and TLR-IL-1R (Toll-like receptor-interleukin type 1 receptor) pathways, respectively. Shown in brackets are known mammalian homologs to Drosophila components of these pathways. Steps that are mediated by unidentified factors are indicated using question marks. Abbreviations: ANK, ankyrin domain; DD, death domain; DED, death effector domain; FADD, Fas-associated death-domain-containing protein; IKK, I-kB kinase; IRAK, IL-1R associated kinase; PGRP, peptidoglycan recognition protein; Rel, REL homolog domain, RIP, receptor-interacting protein; TAK1, transforming growth factor $\beta$ (TGF- $\beta$ )-activated-kinase; TIR, Toll-IL-1R domain.

that constitute a powerful readout to follow the course of the infection [23,24]. Recently, the combination of this latter approach with natural methods of infection has revealed that antimicrobial peptide synthesis is not restricted to the fat-body but can also be induced locally by surface epithelia after contact with incoming microbes [23-25].

Two different methods of infection have been developed to exploit the power of Drosophila genetics in the analysis of host-pathogen interactions. The first method is injection, which involves either pricking the body cavity of the insect with a sharp needle that has been dipped in bacteria or microinjection of a precise dose of microbes directly into the body cavity (Figure 2a). Because the direct introduction of any microbe into the body allows the invading microorganism to be detected, this method of infection always leads to the activation of an immune response that is specific for the class of microbe injected. This method 


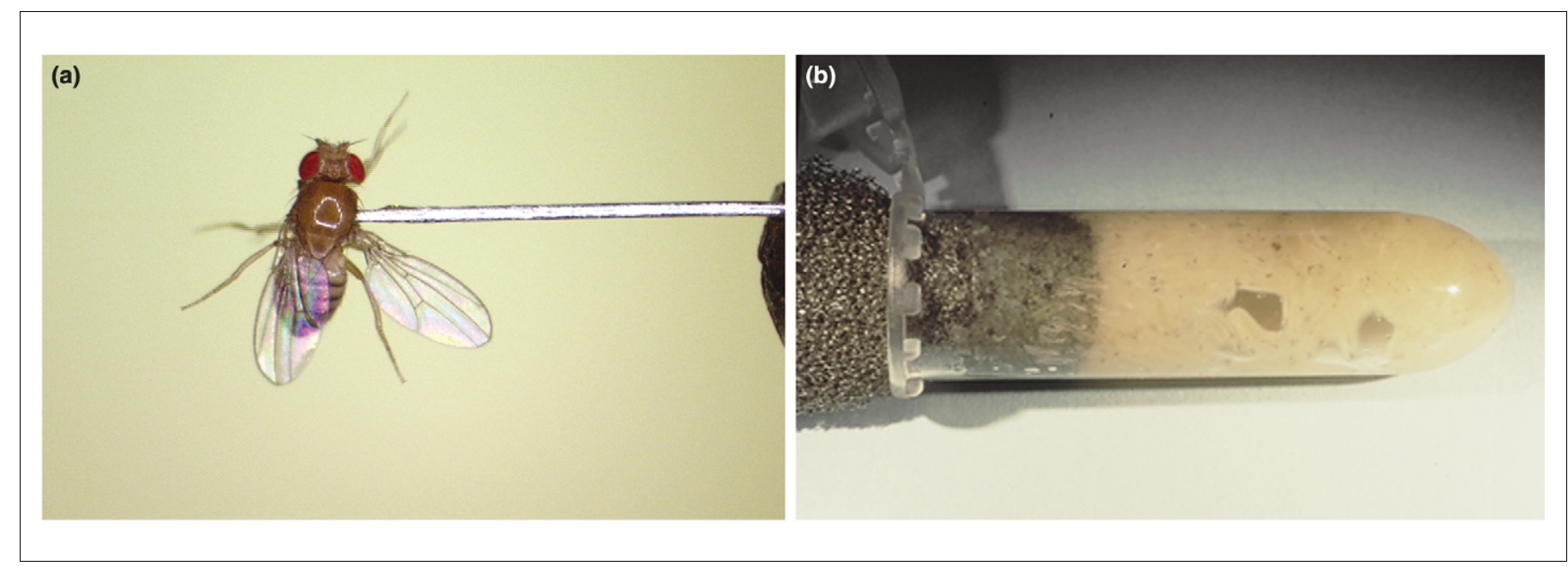

Figure 2. Methods developed to infect Drosophila. (a) Bacterial injection is achieved by pricking adult flies in the thorax with a sharp needle that has been dipped into a concentrated bacterial solution or by microinjecting a precise dose of microbes into the body cavity. Through this type of infection, all microbes induce a strong immune response that is specific for that type of microbe as they are in direct contact with immune system sensors. (b) Natural infection of Drosophila larvae. To mimic the larval natural environment consisting of decaying fruit that is colonized by microbes, larvae are mixed with a solution of crushed banana containing $\sim 10^{11}$ bacteria per ml. To date, only one strain has been shown to induce an immune response using this method, revealing a specific interaction between the two protagonists. Adapted, with permission, from [59], Academic Press.

could also serve as a possible screen for examining pathogenicity because Drosophila recovery from systemic infection depends on both the microbe and the genetic background of the flies. For example, following injection into Drosophila, Escherichia coli, Micrococcus luteus or Aspergillus fumigatus are not pathogenic for wild-type flies; in contrast to Pseudomonas aeruginosa, Enterococcus faecalis or Fusarium oxysporum. The second method of infection, known as natural infection, consists of either feeding Drosophila larvae or adults with a concentrated bacterial solution that has been mixed with their food $[26,27]$ (Figure 2b) or spraying fungal spores directly onto the fly exoskeleton [28]. To date, only a few microbes have been shown to induce an immune response following natural infection $[26,28]$ suggesting that additional specific interactions are required that are not revealed using the injection method.

\section{The post-genomic era}

Understanding the molecular dialogue between a host and its pathogen necessitates the identification of host factors that modulate the fate of the microbe. This can be achieved by the application of heritable RNA interference (RNAi) technology to Drosophila, which allows the generation of directed mutants [29]. Drosophila lines that conditionally express dsRNAs of interest greatly facilitate the silencing of host gene expression. In particular, the role of host factors that have previously been identified as immune responsive genes after bacterial challenge using microarray analyses [19-22] could be assessed using RNAi methodology. Because the Drosophila genome is fully annotated, in vitro dsRNA has been synthesized for every one of its genes [30]; more details can be found at the Drosophila RNAi Screening Center (http://www.flyrnai. org). Coupling RNAi technology with cell-culture-based models enables a functional genome-wide analysis of cellular processes using high-throughput screening to be carried out [30]. This methodology could be applied to analyze the influence of host factors that modulate the fate of bacteria using appropriate readouts, such as cytoskeleton rearrangement, intracellular localization, or bacterial or cellular survival.

\section{Drosophila as a host to identify bacterial virulence factors}

Human opportunistic pathogens: Pseudomonas aeruginosa and Serratia marcescens

Opportunistic pathogens are microbes that infect humans afflicted by specific genetic disorders (e.g. cystic fibrosis) or humans that have impaired defense mechanisms or physical barriers that have been breached (e.g. third-degree burns). They are ubiquitous, versatile bacteria that have developed the ability to adapt to a large number of different environmental conditions. Among them, $P$. aeruginosa and Serratia marcescens are opportunistic pathogens of major impact, and have been shown to be responsible for severe nosocomial infections and, in the case of $P$. aeruginosa, for life-threatening chronic lung infection in cystic fibrosis patients. Their inherent resistance to many antibiotic classes [31,32] constitutes a major clinical problem. This highlights the importance of characterizing their arsenal of virulence factors that are required for pathogenesis with the aim of defining new antibiotic targets. The study of these microbes in mammalian host models requires the development of conditions that mimic the clinical status of infected patients. Several mouse models have been developed but they are expensive and time-consuming. Recent studies have shown that these bacteria have a broad host range and are able to infect invertebrate hosts $[11,13,16,17,33,34]$. These studies have revealed that $P$. aeruginosa uses many of the same virulence mechanisms and effectors in both vertebrate and invertebrate hosts. Different studies have shown that $P$. aeruginosa virulence determinants that are known to be important for mammalian pathogenesis, such as global transcriptional regulators, type III secreted effectors or genes involved in quorum-sensing [27,34-36], were involved in fly death (Table 1). In these studies, 
Table 1. Pseudomonas aeruginosa virulence genes of known function required for full virulence in Drosophila model ${ }^{\mathrm{a}}$

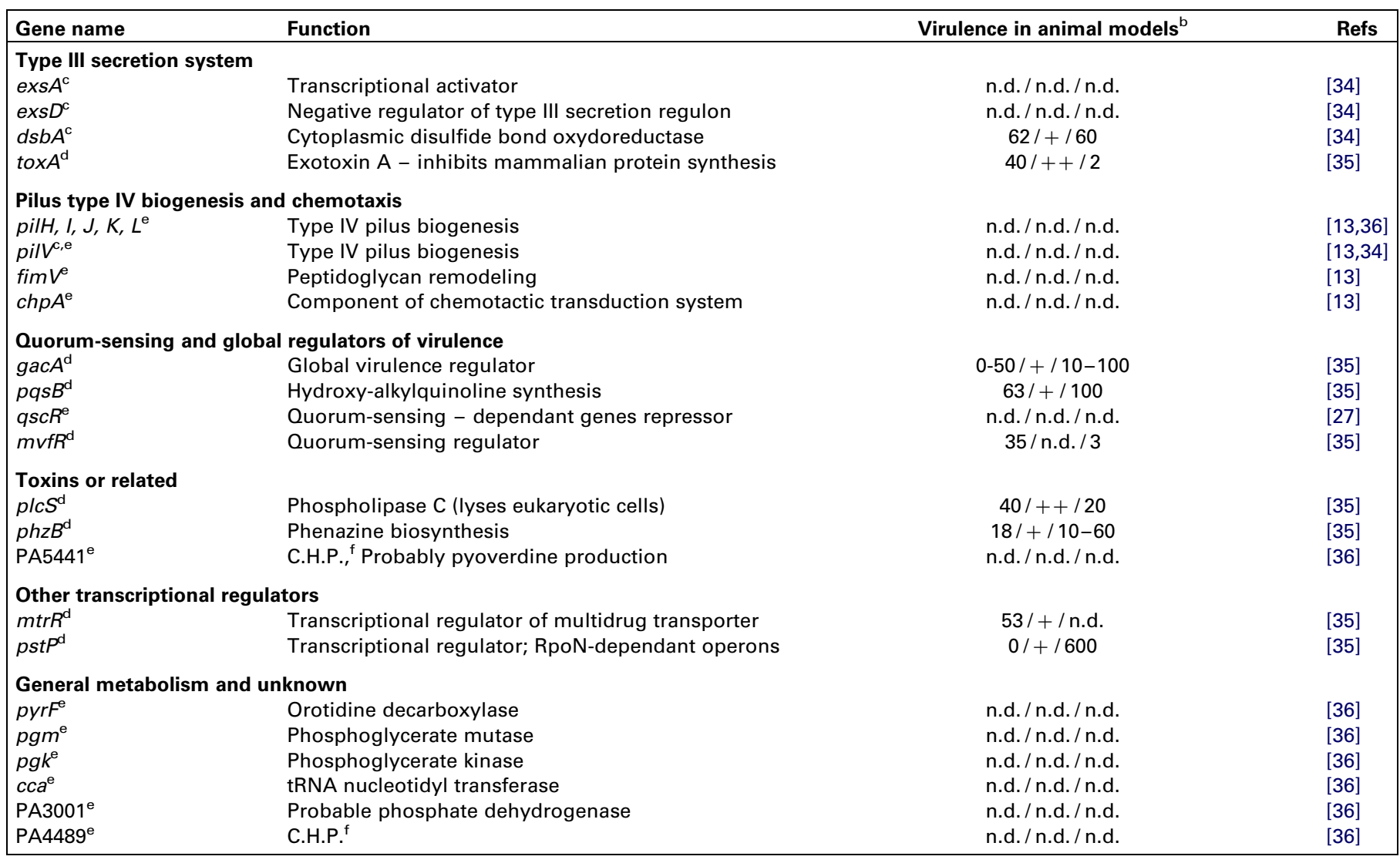

${ }^{a}$ More information, including GenBank accession numbers, is available at the Pseudomonas website (http://www.pseudomonas.com).

${ }^{b}$ Burnt mouse \% lethality (wild-type strain 100\%)/Caenorhabditis elegans killing (++, wild-type; +, reduced) [33]/Galleria mellonella LD50 (wild-type 1) [17]; n.d., data not available. Some gene names have been updated from the date of first publication [60].

${ }^{c}$ Pseudomonas aeruginosa CHA strain.

${ }^{\mathrm{d} P A} 14$ strain.

ePA01 strain.

${ }^{f}$ C.H.P., conserved hypothetical protein.

Drosophila was used to test bacterial mutants after a primary screen had been carried out in other organisms. Another study has reported the use of Drosophila to screen a library of $P$. aeruginosa genetic variants [13], which led to the $P$. aeruginosa pil-chp transduction system being identified as playing an important role in virulence, but its role in a mammalian model remains to be confirmed. Similar studies have been carried out with $S$. marcescens using a multiple-host approach [37]. In this report, the authors first screened $C$. elegans for virulence factors before testing clones that were impaired in virulence in other invertebrate and mammalian models. In addition, it was shown that most of the genes found involved in $S$. marcescens virulence have counterparts in P. aeruginosa, in which they also play an important role in virulence. Altogether, these studies have revealed the existence of conserved virulence mechanisms among opportunistic pathogens that will eventually define the basic requirements for bacterial virulence.

Even though the requirement for some virulence factors depends on the host [37,38], invertebrate models provide a powerful tool to reveal new aspects of bacterial pathogenesis. These examples show that the use of Drosophila as a model host provides novel insights into the identification of both host and pathogen factors that either enhance or restrict bacterial pathogenesis.

\section{Mycobacterium marinum: a macrophage-hidden} bacterium

Mycobacterium tuberculosis causes severe respiratory infections in humans, however, it is difficult to work with established models of mycobacterial infection. Mycobacterium marinum is an appropriate mycobacterial model because it is closely related to $M$. tuberculosis and displays pathogenic properties in its natural hosts (fish and frogs) that are similar to those of M. tuberculosis in humans. In both cases, the bacteria escape from the host immune system by hiding in macrophages. Notably, mutants of $M$. marinum that are impaired in macrophage survival can be complemented using homologous genes from $M$. tuberculosis [39], suggesting that these two bacteria share similar virulence mechanisms. Recently, Dionne et al. [40] showed that injection of $M$. marinum expressing GFP into flies resulted in the accumulation of bacteria within hemocytes. This study showed that after injection the tagged bacteria localized in hemocytes, suggesting a macrophage tropism. In hemocytes that were recovered by bleeding, internalized $M$. marinum did 
not co-localize with either acidified organelles or internalized dead $E$. coli, suggesting effective subversion of phagosome maturation similar to that described for vertebrates. Altogether, these results with $M$. marinum indicate that Drosophila is a promising in vivo model to study bacteria that use macrophages as host cells and has the potential to be applied to other bacteria that escape host defenses by hiding out in macrophages.

\section{Use of Drosophila to identify virulence factor targets}

Another promising approach is the conditional expression of microbial factors in Drosophila that are normally injected into host cells through type III secretion systems; this approach might permit the identification of host factors that are targeted by the type III effector proteins. Type III secretion of virulence factors is a common strategy used by different classes of pathogens to interfere with the cell physiology [41]. In vivo genetic studies have been performed where the type III secreted protein is expressed in the yeast Saccharomyces cerevisiae system to determine their function [42]. Owing to the recent developments in fly genetics, it is possible to express genes in a spatiotemporal-dependent manner in non-vital, easy to screen organs, such as the eye. As observed with the HIV accessory protein Vpu (S. Netter, pers. commun.), conditional expression of type III effectors in the eye might lead to morphological modifications. By crossing such transgenic lines with mutant collections, it might be possible to identify genes that suppress or reinforce these phenotypes, therefore revealing genetic interactions. Biochemical and genetic approaches have been successfully applied in Drosophila to identify and study targets of HIV accessory proteins $[43,44]$.

\section{Drosophila as a host in natural bacterial infections}

The Drosophila injection model has been shown to be relevant for identifying pathogen virulence factors that are important for mammalian infection. However, this method bypasses the first steps of the infection process. Therefore, isolation of the bacteria that elicit a Drosophila immune response through natural routes of infection might reveal strategies that are used by microbes to persist in their natural host. Deciphering host-pathogen interactions in such a way will provide new insights into the initial stages of infection. Because virulence pathways appear to be conserved [45], this will help generate an integrated model for host-pathogen interactions.

Drosophila live in an environment full of microorganisms. However, only a few microbes are known either to trigger the immune response [26] or to be pathogenic [27,46] in Drosophila following ingestion. Among pathogenic strains, the $q s c R$ mutant of $P$. aeruginosa kills Drosophila adults faster than the parental strain, apparently by constitutively expressing several quorum-sensing regulated genes [27]. However, induction of the immune response has not been reported.

Using Drosophila lines expressing a diptericin-gfp reporter gene that reports a Gram-negative inducible response, Basset et al. [26] isolated bacterial strains of Erwinia carotovora spp. carotovora (also called Pectobacterium carotovorum) that are able to induce a strong immune response [26]. E. carotovora are phytopathogenic Gram-negative bacteria of agronomic interest as these bacteria are responsible for fruit soft rot. Among Erwinia strains, several induce a strong immune response in Drosophila larvae by natural infection but are not lethal to their host. It is now possible to apply genetic and genomic approaches on both partners to dissect this 'model' interaction. A genetic screen that was carried out with one of these E. carotovora strains, Ecc15, identified a single gene (evf) that is required for this interaction [47]. Importantly, transfer of evf to several Gram-negative bacteria increases bacterial persistence within the Drosophila gut and leads to the induction of immune responses. The evf gene is regulated by hor, a general regulator of virulence in several Gram-negative bacteria [48]. In Ecc15, hor is required for the infection of both Drosophila and plants, whereas evf was found only in E. corotovora strains that are able to infect Drosophila [47]. These results suggest that bacteria might acquire virulence factors and integrate them into pre-existing regulatory networks, which might constitute a potent evolutionary mechanism to broaden host range.

Finally, Drosophila is known to be a potential vector of bacterial pathogens [49]. The Drosophila-Ecc15 interaction revealed, for the first time, the genetic basis of bacterial persistence in its fly vector. By contrast, the evf gene was not found in other Erwinia strains that are infectious in Drosophila, suggesting that parallel strategies have been selected to allow longer persistence to occur in Drosophila.

\section{Concluding remarks}

The challenge of deciphering host-pathogen interactions is to understand both counterparts in this complex process. Identifying microbial factors required for in vivo virulence has been facilitated by the development of powerful genetic methodologies. Comparative genomics of bacterial pathogens will also give clues about conserved pathways and tropism specificity. Combined with genetic and functional data, it will allow the development of a general model of the role of various virulence genes during infection. The next step will be to build an integrated network of these genes and to understand their role and interplay in the complex process of infection. Such a network would allow the identification of general mechanisms that could be targets of new antimicrobial therapies. Invertebrate models constitute a promising alternative to mammalian models to carry out such analyses. Among these, Drosophila possesses induce defense mechanisms that are similar to those of mammals with respect to both the regulatory pathways involved and the spectrum of their effectors. Moreover, Drosophila is amenable to both forward and reverse genetics, making it a powerful genetic model to study host-pathogen interactions. The study of naturally infectious bacterial strains that are genetically related to human pathogens could highlight the role of virulence factors in the physiopathology of infection. Concerted efforts on the physiopathology of fly infection beyond the immune response would broaden the range of phenotypes and biological processes that can be analyzed. 


\section{Acknowledgements}

We thank Sophie Netter and Carolyn R. Stenbak for critical reading of the manuscript and helpful discussions, and also Bénédicte Devaux, Sébastien Pili-Floury, Christopher Scherfer and Michel Vodovar for critical comments on the manuscript. C.A. is supported by a doctoral fellowship from Conacyt-Sfere (Mexico). Part of this work was supported by the Association de Recherche sur le Cancer (ARC), the Fondation pour la Recherche Médicale (FRM) and a PRMMIP grant.

\section{References}

1 Wilson, J.W. et al. (2002) Mechanisms of bacterial pathogenicity. Postgrad. Med. J. 78, 216-224

2 Lecuit, M. and Cossart, P. (2002) Genetically-modified-animal models for human infections: the Listeria paradigm. Trends Mol. Med. 8, $537-542$

3 Klose, K.E. (2000) The suckling mouse model of cholera. Trends Microbiol. 8, 189-191

4 Shea, J.E. et al. (2000) Signature-tagged mutagenesis in the identification of virulence genes in pathogens. Curr. Opin. Microbiol. $3,451-458$

5 Slauch, J.M. and Camilli, A. (2000) IVET and RIVET: use of gene fusions to identify bacterial virulence factors specifically induced in host tissues. Methods Enzymol. 326, 73-96

6 Rahme, L.G. et al. (1997) Use of model plant hosts to identify Pseudomonas aeruginosa virulence factors. Proc. Natl. Acad. Sci. U. S. A. $94,13245-13250$

7 Plotnikova, J.M. et al. (2000) Pathogenesis of the human opportunistic pathogen Pseudomonas aeruginosa PA14 in Arabidopsis. Plant Physiol. 124, 1766-1774

8 Aballay, A. and Ausubel, F.M. (2002) Caenorhabditis elegans as a host for the study of host-pathogen interactions. Curr. Opin. Microbiol. 5 , 97-101

9 Kurz, C.L. and Ewbank, J.J. (2003) Caenorhabditis elegans: an emerging genetic model for the study of innate immunity. Nat. Rev. Genet. 4, 380-390

10 Cosson, P. et al. (2002) Pseudomonas aeruginosa virulence analyzed in a Dictyostelium discoideum host system. J. Bacteriol. 184, 3027-3033

11 Pukatzki, S. et al. (2002) The human pathogen Pseudomonas aeruginosa utilizes conserved virulence pathways to infect the social amoeba Dictyostelium discoideum. Proc. Natl. Acad. Sci. U. S. A. 99 , 3159-3164

12 Tzou, P. et al. (2002) How Drosophila combats microbial infection: a model to study innate immunity and host-pathogen interactions. Curr. Opin. Microbiol. 5, 102-110

13 D'Argenio, D.A. et al. (2001) Drosophila as a model host for Pseudomonas aeruginosa infection. J. Bacteriol. 183, 1466-1471

14 Brennan, M. et al. (2002) Correlation between virulence of Candida albicans mutants in mice and Galleria mellonella larvae. FEMS Immunol. Med. Microbiol. 34, 153-157

15 Daborn, P.J. et al. (2002) A single Photorhabdus gene, makes caterpillars floppy (mcf), allows Escherichia coli to persist within and kill insects. Proc. Natl. Acad. Sci. U. S. A. 99, 10742-10747

16 Miyata, S. et al. (2003) Use of the Galleria mellonella caterpillar as a model host to study the role of the type III secretion system in Pseudomonas aeruginosa pathogenesis. Infect. Immun. 71, 2404-2413

17 Jander, G. et al. (2000) Positive correlation between virulence of Pseudomonas aeruginosa mutants in mice and insects. J. Bacteriol. $182,3843-3845$

18 Hoffmann, J.A. and Reichhart, J.M. (2002) Drosophila innate immunity: an evolutionary perspective. Nat. Immunol. 3, 121-126

19 De Gregorio, E. et al. (2001) Genome-wide analysis of the Drosophila immune response by using oligonucleotide microarrays. Proc. Natl. Acad. Sci. U. S. A. 98, 12590-12595

20 Irving, P. et al. (2001) A genome-wide analysis of immune responses in Drosophila. Proc. Natl. Acad. Sci. U. S. A. 98, 15119-15124

21 De Gregorio, E. et al. (2002) The Toll and Imd pathways are the major regulators of the immune response in Drosophila. EMBO J. 21, $2568-2579$

22 Boutros, M. et al. (2002) Sequential activation of signaling pathways during innate immune responses in Drosophila. Dev. Cell 3, 711-722

23 Ferrandon, D. et al. (1998) A drosomycin-GFP reporter transgene reveals a local immune response in Drosophila that is not dependent on the Toll pathway. EMBO J. 17, 1217-1227
24 Tzou, P. et al. (2000) Tissue-specific inducible expression of antimicrobial peptide genes in Drosophila surface epithelia. Immunity 13, $737-748$

25 Onfelt Tingvall, T. et al. (2001) The imd gene is required for local Cecropin expression in Drosophila barrier epithelia. EMBO Rep. 2, 239-243

26 Basset, A. et al. (2000) The phytopathogenic bacteria Erwinia carotovora infects Drosophila and activates an immune response. Proc. Natl. Acad. Sci. U. S. A. 97, 3376-3381

27 Chugani, S.A. et al. (2001) QscR, a modulator of quorum-sensing signal synthesis and virulence in Pseudomonas aeruginosa. Proc. Natl. Acad. Sci. U. S. A. 98, 2752-2757

28 Lemaitre, B. et al. (1997) Drosophila host defense: differential induction of antimicrobial peptide genes after infection by various classes of microorganisms. Proc. Natl. Acad. Sci. U. S. A. 94, 14614-14619

29 Ueda, R. (2001) RNAi: a new technology in the post-genomic sequencing era. J. Neurogenet. 15, 193-204

30 Kiger, A. et al. (2003) A functional genomic analysis of cell morphology using RNA interference. J. Biol. 2, 27

31 Stock, I. et al. (2003) Natural antibiotic susceptibility of strains of Serratia marcescens and the S. liquefaciens complex: S. liquefaciens sensu stricto, S. proteamaculans and S. grimesii. Int. J. Antimicrob. Agents 22, 35-47

32 Livermore, D.M. (2002) Multiple mechanisms of antimicrobial resistance in Pseudomonas aeruginosa: our worst nightmare? Clin. Infect. Dis. 34, 634-640

33 Mahajan-Miklos, S. et al. (1999) Molecular mechanisms of bacterial virulence elucidated using a Pseudomonas aeruginosa-Caenorhabditis elegans pathogenesis model. Cell 96, 47-56

34 Fauvarque, M.O. et al. (2002) Role and activation of type III secretion system genes in Pseudomonas aeruginosa-induced Drosophila killing. Microb. Pathog. 32, 287-295

35 Lau, G.W. et al. (2003) The Drosophila melanogaster toll pathway participates in resistance to infection by the gram-negative human pathogen Pseudomonas aeruginosa. Infect. Immun. 71, 4059-4066

36 Potvin, E. et al. (2003) In vivo functional genomics of Pseudomonas aeruginosa for high-throughput screening of new virulence factors and antibacterial targets. Environ. Microbiol. 5, 1294-1308

37 Kurz, C.L. et al. (2003) Virulence factors of the human opportunistic pathogen Serratia marcescens identified by in vivo screening. EMBO J. $22,1451-1460$

38 Hendrickson, E.L. et al. (2001) Differential roles of the Pseudomonas aeruginosa PA14 rpoN gene in pathogenicity in plants, nematodes, insects, and mice. J. Bacteriol. 183, 7126-7134

39 Gao, L.Y. et al. (2003) Transposon mutagenesis of Mycobacterium marinum identifies a locus linking pigmentation and intracellular survival. Infect. Immun. 71, 922-929

40 Dionne, M.S. et al. (2003) Drosophila melanogaster is a genetically tractable model host for Mycobacterium marinum. Infect. Immun. 71, $3540-3550$

41 DeVinney, I. et al. (2000) Phosphatases and kinases delivered to the host cell by bacterial pathogens. Trends Microbiol. 8, 29-33

42 Von Pawel-Rammingen, U. et al. (2000) GAP activity of the Yersinia YopE cytotoxin specifically targets the Rho pathway: a mechanism for disruption of actin microfilament structure. Mol. Microbiol. 36, 737-748

43 Battaglia, P.A. et al. (2001) A Drosophila model of HIV-Tat-related pathogenicity. J. Cell Sci. 114, 2787-2794

44 Leulier, F. et al. (2003) Directed expression of the HIV-1 accessory protein Vpu in Drosophila fat-body cells inhibits Toll-dependent immune responses. EMBO Rep. 4, 976-981

45 Keen, N. et al. (2000) Pathogens and hosts: the dance is the same, the couples are different. Proc. Natl. Acad. Sci. U. S. A. 97, 8752-8753

46 Flyg, C. et al. (1980) Insect pathogenic properties of Serratia marcescens: phage-resistant mutants with a decreased resistance to Cecropia immunity and a decreased virulence to Drosophila. J. Gen. Microbiol. 120, 173-181

47 Basset, A. et al. (2003) A single gene that promotes interaction of a phytopathogenic bacterium with its insect vector, Drosophila melanogaster. EMBO Rep. 4, 205-209

48 Thomson, N.R. et al. (1997) The rap and hor proteins of Erwinia, Serratia and Yersinia: a novel subgroup in a growing superfamily of 
proteins regulating diverse physiological processes in bacterial pathogens. Mol. Microbiol. 26, 531-544

49 Janisiewicz, W.J. et al. (1999) Fate of Escherichia coli O157:H7 on fresh-cut apple tissue and its potential for transmission by fruit flies. Appl. Environ. Microbiol. 65, 1-5

50 Rong, Y.S. and Golic, K.G. (2000) Gene targeting by homologous recombination in Drosophila. Science 288, 2013-2018

51 Rubin, G.M. and Spradling, A.C. (1982) Genetic transformation of Drosophila with transposable element vectors. Science 218, 348-353

52 Brand, A.H. and Perrimon, N. (1993) Targeted gene expression as a means of altering cell fates and generating dominant phenotypes. Development 118, 401-415

53 Duffy, J.B. (2002) GAL4 system in Drosophila: a fly geneticist's Swiss army knife. Genesis 34, 1-15

54 Kennerdell, J.R. and Carthew, R.W. (2000) Heritable gene silencing in Drosophila using double-stranded RNA. Nat. Biotechnol. 18, $896-898$

$55 \mathrm{Xu}, \mathrm{T}$. and Rubin, G.M. (1993) Analysis of genetic mosaics in developing and adult Drosophila tissues. Development 117, 1223-1237

56 Adams, M.D. et al. (2000) The genome sequence of Drosophila melanogaster. Science 287, 2185-2195

57 Celniker, S.E. et al. (2002) Finishing a whole-genome shotgun: release 3 of the Drosophila melanogaster euchromatic genome sequence. Genome Biol 3 RESEARCH0079.1-0079.14 (http://genomebiology.com/)

58 Giot, L. et al. (2003) A protein interaction map of Drosophila melanogaster. Science 302, 1727-1736

59 Tzou, P. et al. (2002) Methods for studying infection and immunity in Drosophila. In Methods in Microbiology (Vol. 31) (Sansonetti, P.J. and Zychlinsky, A., eds), pp. 507-530, Academic Press

60 Tan, M.W. (2002) Cross-species infections and their analysis. Annu. Rev. Microbiol. 56, 539-565

\section{Microbiology websites}

\section{The Microbiology information portal}

http://www.microbes.info/

A useful website providing a variety of microbiology links, including links to feature articles and microbiology-related news stories.

\section{All the virology on the WWW}

http://www.tulane.edu/ dmsander/garryfavweb.html

A comprehensive website on virology, including great pictures, virology jobs, a bookshop and links to other useful sites.

\section{The Picornavirus homepage}

http://www.iah.bbsrc.ac.uk/virus/Picornaviridae/

A website dedicated to the family of the Picornaviridae. Includes important up-to-date news features and articles, ongoing research at the Institute for Animal Health, comprehensive virus classification, links to other useful websites and details of future EUROPIC conferences.

\section{The WWW Virtual Library: Mycology}

http://biodiversity.bio.uno.edu/ fungi/

A useful website that provides extensive links to other related sites.

\section{The World Wide Web Virtual Library: Parasitology}

http://www.diplectanum.dsl.pipex.com/purls/

A website that provides extensive links to other related sites.

\section{Parasitology links}

http://www.galenica.cl/club/rec_parasitologia.html

\section{The world of parasites}

http://martin.parasitology.mcgill.ca/JIMSPAGE/WORLDOF.HTM

Find out what parasites live with you in your country!

\section{The aspergillus website}

http://www.aspergillus.man.ac.uk/

This site includes laboratory protocols, treatment information, DNA sequence data, a comprehensive bibliographic database, an image library and discussion groups.

\section{The $E$. coli index}

http://web.bham.ac.uk/bcm4ght6/res.html

A comprehensive guide to information relating to the model organism Escherichia coli.

Bacterial infections and mycoses 